\title{
Educación integral en Santo Tomás de Aquino*
}

\section{Jaime Ricardo Reyes Calderón**}

Recibido: 2 de agosto de 2015 - Aprobado: 8 de septiembre de 2015

\section{Resumen}

El presente ensayo quiere volver los pasos de la reflexión educativa sobre una fuente fundamental en la historia de la filosofía de la educación: el planteamiento de Santo Tomás de Aquino. Quien constituye un avance formidable en la evolución del concepto acerca de lo humano, superando una tradición neoplatónica que había desvirtuado la realidad humana y también el horizonte de salvación religiosa cristiana. Con Santo Tomás se desarrolla un criterio diferenciador y crítico de lo educativo: el concepto de educación integral.

Palabras clave: antropología, educación integral, Santo Tomás, virtud.

* El artículo es resultado de la investigación especializada del autor. DOI: http://dx.doi. org/10.15332/s2011-9771.2016.0001.03

** Lic. en Filosofía, Esp. en literatura, Esp. en desarrollo humano, Magíster en Educación, Candidato a Doctor en Educación. Coordinador de la Institución Educativa Santo Ángel de Cúcuta. Profesor Asociado de la Universidad Libre de Cúcuta. Correo electrónico: jaime.reyes@ unilibrecucuta.edu.co 


\title{
Comprehensive education in St. Thomas Aquinas
}

\begin{abstract}
This essay wants to return the steps of educational thinking about a vital source in the history of the philosophy of education: the approach of Saint Thomas Aquinas. Aquinas is a formidable advancement in the evolution of the concept about human, overcoming a neoplatonic tradition that had distorted the human reality and also the horizon of religious salvation Christian. Santo Tomás develops a distinguishing and critical criterion of education: the concept of integral education.
\end{abstract}

Keywords: Anthropology, education, Saint Thomas, virtue.

\section{Éducation intégrale à Saint Thomas D’Aquin}

\section{Résumé}

Cet essai veut revenir sur les traces de la réflexion éducative sur une source fondamentale dans l'histoire de la philosophie de l'éducation: l'approche de Saint Thomas d'Aquin. Celle-ci constitue un progrès formidable dans l'évolution du concept a propos de l'humain, surpassant une tradition néo-platonnienne qui avait affaibli la réalité humaine et l'horizon de la rédemption religieuse chrétienne. Avec Saint Thomas d'Aquin un critère différentiant et critique de l'éducatif se développe: le concept d'éducation intégrale.

Mots-clés: Anthropologie, éducation intégrale, Saint Thomas, vertu. 


\section{Introducción}

El Doctor Angélico insiste en un rasgo esencial definidor del sentido educativo: el respeto a la dignidad de la persona. Más allá de las épocas, las intenciones morales y religiosas, las mentalidades políticas y el horizonte cultural; el quehacer educativo se constituye en la preocupación por superar los límites de lo físico, lo material, lo aparentemente obvio y primario, para enfatizar una complementariedad en la que el discurso salvífico no relega lo humano a simples funciones concretas y exterioristas.

En la época medieval donde el eje de comprensión de la realidad era lo religioso, las relaciones con el trascendente, si se quiere, mandato evangélico y credo educativo personalista, indican que lo humano significa algo espiritual y cualitativamente distinto, y que tal peculiaridad es precisamente el carácter que lo diferencia del resto de los seres, o del resto de presencias existenciales. Recordemos que para la mentalidad medieval existían los cuerpos, las almas, las almas en pena, las almas purificadas, los ángeles y los santos de Dios. Hablar del hombre, desde Santo Tomás, era hablar de la persona, no de las corporalidades, o las dignidades nobiliarias, o las determinaciones psicometafísicas tan propias del platonismo. En Tomás "Es así como, de entre todas las sustancias, los singulares de naturaleza racional tienen un nombre especial. Este nombre es persona." (Aquino, 2001, p. 322).

Así, Santo Tomás fue gestor de una gran revolución del pensamiento en el siglo XIII. Hizo parte del descubrimiento de Aristóteles agenciado por los sabios árabes, en oposición a la tradición de la Iglesia que se había mantenido fiel, por casi mil años, a las posturas neoplatónicas derivadas del agustinianismo. Gilson, a propósito de la división medieval entre neoplatónicos y neoaristotélicos, sintetizará: "Siempre ha habido, y quizá siempre habrá, espíritus que consideren al platonismo como una filosofía naturalmente cristiana y al aristotelismo como la filosofía natural de los paganos" (1952/1999, p. 73). Los rasgos de crítica, investigación y reinvención, hacen parte del espíritu tomista que en sus fuentes mostró valentía e independencia al apartarse de lo establecido. Tal confrontación, que significó también una cierta pugna entre dos grandes y gloriosas órdenes religiosas, los franciscanos y los dominicos, arrojó como resultado lamentable la condena de muchas de las proposiciones y documentos salidos de la mano del Doctor Angélico.

Un segundo gran rasgo de la filosofía tomista, consecuencia del cambio de mentalidad gestado por Tomás, es la recuperación de la materialidad, la 
sensibilidad, la corporalidad, en la consideración del hombre, el conocimiento y la moral. "Por primera vez en la historia de la cristiandad un teólogo trató de armonizar plenamente la experiencia sensorial con la inteligencia y los requerimientos de la fe, y de hacer justicia a ambos" (Bowen, 1992, pp. 212-213). Con esto cesa la condena que la tradición impuso al ejercicio de lo sensible y corporal.

La educación en nuestro mundo actual, en nuestro contexto colombiano, en lo atinente a la formación profesional, debe ofrecer elementos para la formación de competencias del ciudadano colombiano, bajo el fin de realizar una formación integral, que sería el primer gran objetivo de la educación superior según la Ley 30. Para aportar elementos que enriquezcan el sentido de integralidad, en este artículo de reflexión se responderá a la gran pregunta ¿qué es educación integral?, asumiendo como respuesta las líneas identificadas en Santo Tomás de Aquino. En este estudio seguimos un método hermenéutico, de investigación documental, a través de la técnica del comentario de texto. En un primer momento, recordamos las dimensiones del ser humano según Santo Tomás, para posteriormente definir la integralidad desde el horizonte prescrito por Santo Tomás en los términos de una educación para la perfección, una educación eminentemente ética fundada en las virtudes.

\section{Dimensiones del ser humano}

En Santo Tomás el ser humano resulta una correlación de tres dimensiones: la vegetativa-orgánica, la animal-sensitiva y la racional-intelectiva. Un primer nivel del ser humano lo podemos denominar orgánico. El hombre experimenta unos apetitos naturales propios de la nutrición, el desarrollo y la generación. Estamos ante la consideración de la vida vegetativa, que permite la subsistencia de la materialidad corporal humana, sin concurso ni de afectos, ni de inteligencias: "La nutrición, el desarrollo y la generación cumplen con lo que en el hombre es la vida vegetativa". (Beuchot, 2004: 171). Santo Tomás afirma que la sociedad debe asegurar los medios para que estas funciones sean satisfechas de forma básica, de suerte que se provea la subsistencia de todos.

El segundo nivel del ser humano comparte elementos propios de los seres animales y de lo personal-racional. Estamos en lo sensible. El aquinate diferencia dos tipos de sentidos, los externos y los internos. Además de los sentidos externos (tacto, olfato, gusto, audición, visión), Tomás añade, ya en el campo cognoscitivo, los sentidos internos: el sentido común (que distingue lo propio de cada acto de sentir), la imaginación (la generación de una imagen de lo sentido) 
y la memoria (facultad encargada de almacenar las imágenes). Como esta organización está determinada referencialmente; es decir, opera por afección de lo externo independiente de la voluntad y la libre creatividad humana, constituirán el entendimiento paciente.

No obstante, los apetitos sensibles están precedidos y acompañados de un cierto conocimiento pues persiguen un bien sensible, no son automáticamente inconscientes. Los apetitos sensibles "se dividen en dos especies: el apetito concupiscible y el apetito irascible" (Beuchot, 2004, p. 173). El primero busca lo conveniente y evita lo nocivo. De este apetito se derivan pasiones como amor, odio, deseo, fuga, gozo y tristeza. Los apetitos irascibles incitan a defenderse contra la adversidad, por ello las pasiones que lo representan son la esperanza, la desesperación, la audacia, el temor, la ira.

Siguiendo a Aristóteles, el tercer nivel de lo humano en Santo Tomás enuncia que la cualidad básica del ser humano, su esencia, su "quiddidad", su "forma", aquello que lo hace original en medio de la escala de los seres, es el intelecto agente, la actividad superior de lo racional. Corresponde, como primera perfección de lo racional, la vida teórica. La razón es intelectiva y es raciocinativa. Lo propio del intelecto es conocer las esencias por una aprehensión abstractiva, su objeto es la verdad. La razón raciocinativa tiene como objeto propio el orden pues dispone del acto del intelecto y del acto del juicio para conseguir la verdad. "El intelecto tiene como objeto propio la verdad (esencial-existencial), y la razón tiene como objeto propio el orden" (Beuchot, 2004, p. 174). La verdad de las cosas asumidas en el orden que tal verdad determina, forma el objetivo de la contemplación humana en su aspecto inmanente. Los productos de la esencia racional del hombre son la sabiduría, la ciencia, la prudencia y el arte.

La razón también tiene un lado apetitivo, la voluntad. "La voluntad es el apetito que tiene como objeto el bien conocido por la razón" (Martínez, 2002: p. 275). La voluntad quiere poseer de manera permanente el bien, se inclina naturalmente a la consecución de la felicidad. Por ello debe escoger continuamente aquello contingente que se le presenta y que corresponde convenientemente al fin perseguido. Cuando el hombre permite que la voluntad siga al intelecto, se da entonces la auténtica libertad. La voluntad se manifiesta en la vida práctica del hombre, en sus haceres continuos, por ello su finalidad es la transformación de la realidad externa guiada por el bien.

Razón y voluntad se articulan para actuar, jalonan la consecución del bien, llevan al hombre, en los márgenes de su inalienable libre albedrío, hacia los confines que perfeccionan su ser, su saber y su querer. Así, en Santo Tomás conocimiento, 
voluntad y trascendencia no se pueden desligar. El hombre que conoce es una creatura creada por Dios. Ello también permite asociar los conocimientos finitos de los sentidos a los conocimientos infinitos, trascendentes, propios de otras realidades. El hombre produce saberes según la calidad de los objetos que su alma racional considera. El mayor objeto y consecuentemente, la mayor empresa del ser humano, lo conduciría a Dios. Debido a su alma racional y a su condición creatural, Santo Tomás consigue aunar las bases realistas del conocer aristotélico con la fe cristiana. Veamos esta precisión epistemológica-moral de claro énfasis teológico, de manos del conocido especialista jesuita, F. Copleston (2004):

En esta vida, nuestro conocimiento de Dios es imperfecto y analógico, solamente conocemos a Dios de una manera indirecta, mientras que la voluntad tiende directamente a Dios: el amor a Dios es, pues, más perfecto que el conocimiento de Dios. En el caso de objetos que son menos nobles que el alma, los objetos corpóreos, podemos tener conocimiento inmediato, y tal conocimiento es más perfecto que la volición; pero en el caso de Dios, un objeto que trasciende al alma humana, solamente tenemos, en esta vida, un conocimiento mediato, y nuestro amor a Dios es más perfecto que nuestro conocimiento de Dios. No obstante, en la visión beatífica, en el cielo, cuando el alma ve la esencia de Dios de un modo inmediato, la superioridad intrínseca del entendimiento sobre la voluntad se reafirma a sí misma, por así decirlo. De esa manera, santo Tomás adopta la actitud intelectualista de Aristóteles y la interpreta, a la vez, en un marco cristiano (Copleston, 2014, pp. 308-309).

El crecimiento en la caracterización de lo humano, dentro de las tradiciones de reflexión católicas, ha acercado a Santo Tomás al personalismo. Recogemos las argumentaciones de Díaz, brillante actualizador de los cánones personalistas, y afirmamos que el hombre en Santo Tomás es un ser privilegiado por su razón, autonomía, libertad y responsabilidad. Seguimos a Díaz, pues, y hacemos nuestras sus precisiones:

Partiendo de la definición de Boecio, definirá a la persona como «individuo de naturaleza intelectual por sí mismo subsistente», y por ello como «el ser perfectísimo en toda la naturaleza». Su dignidad y perfección ontológica está en existir por sí con entera independencia, y su dignidad y perfección dinámica está en poseer naturaleza intelectual (o racional, o espiritual), lo que conlleva el dominio de sus propios actos cual ser libre y responsable (Díaz, 2002, p. 27). 
Así pues, a la persona se le ofertan exigencias de realización que desembocan en compromisos con la sociedad. Muy aristotélicamente, recordando que el estagirita definía al ser humano como zoon politikon, como animal político, Tomás conectará la entidad personal con la entidad colectiva: “[A la persona] La voluntad le impone una exigencia de praxis conforme a la razón, una exigencia de trabajo conforme a la naturaleza humana, una exigencia de justicia, que, bien radicada en la voluntad, conlleva una exigencia de amistad o armonía debida a la proporcionalidad" (Beuchot, 2004: p. 176). Para tal efecto, la educación encerrará un claro cometido pues las dificultades individuales entorpecen esa armonía y esa amistad deseada en los planos sociales. La voluntad humana es educable, es en este sentido que se requiere de la educación moral como perfeccionamiento de los hábitos de la voluntad (Reyes, 2010: pp. 22-24).

Ahora bien, todo ser tiende a un fin. El fin que perfecciona al ser humano, la forma suprema que le trae al hombre la más grande felicidad, es la consideración de la causa primera, del Ser por excelencia, inicio y meta de lo creado: Dios.

No obstante, definir creacionalmente al hombre no es restarle iniciativa, no es "aparentar" una individualidad independiente. El hombre es libre y por el manejo razonable y voluntarioso de sus pasiones, hace de sus decisiones actos humanos virtuosos. En Tomás virtud y trascendencia se gestan en los campos de la libertad (Martínez, 2004).

Por esta razón en Santo Tomás el ser humano no es solamente una naturaleza que se agota en los oficios de la reproducción y la nutrición. El hombre y su derrotero substancial racional, se reconoce bajo el término de persona. Así, "Las notas que pertenecen a un individuo en cuanto singular, como los actos personales y las cosas que le son propias, no se transmiten de los padres a hijos" (Aquino, 1989, p. 637). De esta manera, entendemos que el hombre, en cuanto naturaleza universal, posee un alma racional, pero en cuanto alma individual, está revestido de un carácter de unicidad y originalidad que supera los rasgos comunes. La persona es en ella misma, una naturaleza única e irrepetible.

Si el hombre fuera mera materialidad, bastaría el ejercicio de su alma vegetativa y apetitiva. Si el hombre -como lo pretendía Platón-, fuera exclusivamente un alma racional sometida al juego incesante y paradójico de las reencarnaciones, entonces la vida terrena y las empresas temporales y finitas serían una enorme charada. Ambas perspectivas antiguas cercenan la humanidad. Es por esto que en Tomás la vida humana empieza con las situaciones concretas. El cuerpo humano del ser humano, es la perfección esencial. El ser humano real, es el ser que se enfrenta al devenir de las cosas y las circunstancias. $Y$ allí se reconoce el hombre como orientado a múltiples perspectivas. Lo particular, lo sensible, lo de 
todos los días, no puede ser ignorado en una revaloración del hombre como ser de perspectivas y de dimensiones conjugadas.

\section{Educación para la perfección}

Para definir educación en clave tomista, seguimos a Millán Puelles (1989), quien sentencia que: "Educatio, cuyo primer sentido fue el de la alimentación o nutrición, haya pasado a significar la actividad por la que se promueve y favorece el perfeccionamiento de las facultades espirituales del hombre" (Millan-Puelles, 1989, p. 31). Las capacidades del hombre deben ser alimentadas pues:

Al venir al mundo tiene el hombre la inteligencia y la voluntad como dormidas, todavía incapaces de servir a su fin, por falta de desarrollo y madurez. El adquirir la respectiva formación es tan necesario a estas potencias como lo es el alimento al cuerpo (Millán-Puelles, 1989, p. 32).

En Santo Tomás la educación como formación desplegaría el desarrollo del ser humano en la dinámica procreativa (óntica), en la dinámica de subsistencia (nutricional) y en la dinámica disciplinar, racional, cognitiva (instructiva). La educación del ser humano se contextúa en una educación de la virtud, en una educación para la perfección de sus esencias humanas diferenciadoras. Educar no es entrenar para unos gestos corporales o verbales. No es lanzar al hombre a una vocación de supervivencia físico-material. Educar atiende a la persona en su completud, en sus dimensiones, en su complejidad personal e histórico-social. Millán Puelles nos refiere que la educatio, en sentido pleno revestiría entonces tres caracteres:

- Se trata de algo que solo conviene al hombre, cuyo cuerpo lo informa un alma espiritual que también debe ser perfeccionada.

- Conviene al hombre entero y no a una parte o aspecto aislado en él, aunque también es cierto que principalmente se refiera a la formación del alma humana. Lo equivalente a la educación en su sentido puramente espiritual o moral se expresa en los términos instructio y disciplinae.

- Es distinto y complementario de la generación o procreación. La diferencia entre procreación y educación no impide que la segunda complete y perfeccione la primera, que deja al hombre en la necesidad de recibir la ayuda que le permita el desarrollo físico y espiritual para, en su día, poder valerse por sí mismo (Millán, 1989). 
Usando lenguaje de educadores contemporáneos diremos que se quiere representar la actividad humana, su ser y hacer, en un afán de integralidad, de conjugación de las distintas dimensiones del ejercicio que despliega el hombre. Tomás especificará que el ser humano es su hacer (YO), es su obrar (Alteridad), es el intelectivo ejercicio del comprender (Cognición), y es el amplio espacio de su comunicación (Trascendencia) (Restrepo, 2009).

No hay hacer plenificante, perfeccionante, que no se oriente a un fin. La educación no se agota en sí misma, ingresa en el ámbito de lo racional para enaltecer al hombre, para disponer del desarrollo de sus potencias personales. Afirmamos con Beuchot (2004): “¿Cuál es para Santo Tomás la finalidad del magisterio? Es lograr la perfección del "hombre total", del hombre íntegro, del hombre en todo el organismo corporal y espiritual, en todas sus potencialidades y virtudes (Beuchot, 2004, p. 231)".

Millán, acerca del fin de la educación como estado de virtud, analoga el hombre a la madera tallada perfecta, de modo que cumplir bien con un oficio exige la íntima concatenación del bien obrar humano respecto a su libertad y su voluntad. Ahora bien, las virtudes no son una operación desconectada de la realidad circundante, de la percepción del compromiso con los otros. El obrar humano es virtuoso cuando construye relaciones justas en su sociedad. Tales relaciones justas, en el bienestar de todos, hacen posible la aspiración humana a la felicidad. Así, podemos indicar que la educación es también una actividad que procura la plenitud humana en la vivencia personal y comunitaria de la felicidad. "En la misma búsqueda del bien común de la sociedad, y para la convivencia correcta, el hombre necesita de las virtudes; por eso la vida virtuosa es su perfección y ella es también el proceso de la consecución de un fin, su felicidad" (Beuchot, 2004, p. 193).

Nos permitimos un breve rodeo acerca de la educación en la virtud. Virtud es «una cualidad del alma por la cual vivimos rectamente y de la que no puede usarse mal» (Hernanz, 1945, p. 105). Las virtudes constituyen criterios dinámicos, fuerzas humanas que se objetivan en el actuar pero que nacieron naturalmente en el alma racional de la persona. Tomás ofrece dos clases de virtudes, las morales (en donde sobresalen las virtudes cardinales) y las intelectuales, clases análogas a las virtudes éticas y dianoéticas de Aristóteles.

Las virtudes morales, en Aristóteles, hacen parte no del núcleo central, original y natural del ser humano, sino a su contexto comunitario de suerte que respecto a las intelectuales: "la ética, en cambio, procede de la costumbre, como lo indica el nombre que varía ligeramente del de «costumbre». De este hecho resulta claro que ninguna de las virtudes éticas se produce en nosotros por naturaleza, puesto que ninguna cosa que existe por naturaleza se modifica por costumbre" 
(Aristóteles, 1993, p. 158). Las virtudes éticas se ordenan al recto uso de la voluntad para conseguir el bien. Son virtudes que perfeccionan el obrar desde el obrar mismo. Las virtudes morales son la prudencia, la justicia, la templanza y la fortaleza. Estas primeras virtudes se reconocen en la vivencia individual del libre albedrío, haciendo parte de las decisiones y relaciones particulares, concretas y cotidianas.

Por su parte, las virtudes intelectuales se asemejan a las enunciadas en Aristóteles como virtudes dianoéticas. El estagirita bien delimita las posibilidades de estas al sentenciar: "La dianoética se origina y crece principalmente por la ensenanza, y por ello requiere experiencia y tiempo" (Aristóteles, 1993, p. 158). Relacionadas estrechamente con el proceso de formación implicado en la enseñanza, estas corresponden al ejercicio de la inteligencia teórica, ese ver que impulsado por el asombro penetra en las causas últimas de la realidad, dando cuenta de un gozo continuo que acompaña toda experiencia. Aquí nos adentramos en los saberes superiores, se contemplan los objetos que son necesarios y que no admiten ningún tipo de contingencia. Las virtudes intelectuales funcionan desde el centro esencial mismo del ser humano: su razón, principio identificador fundamental. Las virtudes intelectuales pertenecen a la facultad intelectiva, al alma racional esencial de ser humano. Cinco son las virtudes intelectuales: la sabiduría, la ciencia, la inteligencia, el arte y la prudencia. Las tres primeras se ocupan de los dictámenes del entendimiento teórico y las dos últimas del entendimiento práctico.

Luego tanto las virtudes morales (cardinales) como las intelectuales proceden de ciertos principios naturales preexistentes en nosotros. En Santo Tomás ¿Como se cultivarían estas virtudes intelectuales?, el estudioso dominico Hernanz especifica:

La sabiduría se distingue de la ciencia. La ciencia-episteme-es un estar encima: conocer muchas cosas sin desorden, pero sin unidad. En cambio sólo hay una sabiduría porque sólo hay una primera causa. Ese saber de la primera causa es la «sapientia», la ciencia sápida. Porque la sabiduría es el conocimiento supremo, luz que ilumina y vivifica los conocimientos particulares, penetración aguda de todas las cosas por medio de esa visión, perspectiva grandiosa que resulta poética y, artística. Es la síntesis aplicada desde las altas cumbres. Es la filosofía. La metafísica llega a ser entonces algo vivo, y el metafísico, el que conoce lo que las cosas son viviéndolas y haciéndolas vivir en él (Hernanz, 1945, p. 106). 
Al lado de estas tres virtudes (inteligencia, ciencia y sabiduría) que arrancan de hábitos intelectuales especulativos, hay otras dos: el arte y la prudencia, que pertenecen al entendimiento práctico.

De otra parte, un tercer tipo de virtudes, las virtudes teológicas o teologales, a saber: fe, esperanza y caridad, nos son infundidas por Dios (son pues, creacionales, también asociadas a los dones del Espíritu Santo) completando los principios de actuación de las virtudes morales. Estas virtudes agenciadas por la gracia divina se apartan del criterio general del "justo medio" que sostiene la actividad de todas las virtudes y que es además, el contenido decisivo de la prudencia. Lo teologal se distinguiría de la acción de la virtud moral pues: "cuando un hombre valiente teme lo que debe ser temido, es virtuoso, y el no temerlo sería un vicio. Pero cuando, confiado en la ayuda divina, nada teme en este mundo, es sobrehumano. Y las virtudes de este tipo son llamadas divinas" (Copleston, 1960, p. 240). Concluye el doctor angélico: "Es claro por lo dicho (q.62 a.1; q.66 a.4 y 6) que el hombre se ordena a Dios por las virtudes teologales; por la templanza y la fortaleza, a sí mismo; y por la justicia, al prójimo" (Aquino, 2000, p. 564).

En Santo Tomás, podemos colegir, una educación en la virtud tendría que permitir el desenvolvimiento de todas las posibilidades de acción virtuosa. Tendría que ser fundamentalmente un proceso de actualización de las virtudes en cada ser humano, desarrollando entonces lo moral-práctico, lo intelectual-teórico y lo espiritual-teológico. Tal sería la educación que convendría al alma humana, a la persona y sus posibilidades de crecimiento y perfeccionamiento.

La educación en la virtud, la educación que procura la perfección de la persona, es producto entonces de tres factores: el hombre (sí mismo), la sociedad (prójimo) y la gracia (Dios). Los tres factores, que se distinguen para reconocer su origen diverso, en la cotidianidad, en el ejercicio puntual por el cual se evidencian como hábitos de actuaciones virtuosas, son en realidad inseparables: al realizar una decisión, el hombre determinado por su naturaleza racional, individual y original, por sus costumbres sociales y por su caminar como creyente, como creatura de Dios, actúa fundiendo todas las operaciones derivadas de tales fuentes. He aquí los tres medios indispensables para este proceso educativo del hombre, que ha de conducirle hacia la suma perfección y felicidad.

Se dirige la dinámica educativa, moral y racional, hacia el acto educativo conformado por el maestro, el discípulo y lo enseñado. La actividad de educar se ejerce por parte de un maestro quien tendría como función el llevar a un hombre a su perfección. El conocimiento, como todas las esencias creadas, viene de Dios y son infundidas por él en el alma humana. Pero tal acción se completa gracias al 
maestro, continuador de la acción creacional, análogo del motor inmóvil siendo motor de entendimiento, quien aporta su actividad para conseguir que las ciencias y las virtudes, que están en potencia, logren realizarse en acto, en el educando. Con palabras del doctor angélico: “El maestro, consiguientemente, estimula el entendimiento a saber lo que enseña, haciendo las veces de motor esencial que hace pasar de la potencia al acto" (Aquino, 2007, p. 15).

El maestro, además, "engendra espiritualmente" como el padre físico "engendró corporalmente" (Martínez, 2004. p. 80). Fundamentalmente, porque en relación a ese humano en formación, el maestro es modelo de conducta y de saber. Para Beuchot (2004), recordándonos un poco el método mayéutico de Sócrates, en Tomás la acción del maestro, educere, se insiste, es:

Hacer pasar de un estado a otro; en sentido aún más fuerte equivale a sacar o ayudar a salir, mediante una influencia exterior, a una cosa del ámbito de lo meramente posible a lo actual...es perfeccionar, es llevar a un sujeto de sus meras potencialidades a la perfección, mediante la enseñanza (Beuchot, 2004, p. 232).

El compromiso de perfeccionarse personalmente trasciende a la esfera de lo social, de tal suerte que al líder, al regente; se le exige apartarse de las prácticas tiránicas, de manera que su acción política redunde en bienestar de todos. Siguiendo el libro de los Proverbios, Santo Tomás al regente le determina que "el hombre que preside sin razón, llevado de sus pasiones, en nada se diferencia de la bestia" (Del régimen de los príncipes, Libro I, cap. 2), prescribiendo entonces que su ejercicio es una labor absolutamente racional.

\section{Conclusiones}

Con Santo Tomás, la pedagogía debe ser considerada un bien invaluable, un ejercicio que alimenta al niño y al joven en todas sus dimensiones. Es necesario que los planes educativos toquen la nutrición primaria, además. Un pueblo con hambre lo único que aprende es el resentimiento y la violencia revanchista. Un pueblo sin acceso a los bienes culturales estará condenado a repetir los pecados de su historia pasada. De un tiempo para acá, a cualquier capricho le llaman "libre ejercicio de la personalidad". Y entonces las modas, accesorios, las consignas emocionales acerca de lo social, lo decorativo y cosmético; lo carente de raíces profundas en la comprensión de lo real, se vuelven tópicos y hábitos más importantes que 
el desarrollo de las capacidades profundas del ser humano. El hoy pide también que se eduque el control de la voluntad, el uso razonable y virtuoso de la libertad, es decir, una educación para asumir, entender y decidir sobre los núcleos más esenciales de nuestra existencia.

Una educación integral debe afirmar las notas esenciales de la personalidad de cada uno. Debe ser virtuosa en el sentido de perfeccionar cada una de las posibilidades y capacidades de la persona. Debe también ofrecer una dimensión de justicia, de entrega, de solidaridad, de búsqueda del bien común, de respeto y donación al otro que es mi hermano. La integralidad robustece el intelecto como la capacidad de poner en acto las explicaciones que dan coherencia y crecimiento a las relaciones entre los seres. La dimensión cognitiva no se centra exclusivamente en el ejercicio de las ciencias, se dispone también en la toma de decisiones de una conciencia humana libre, responsable, virtuosa, íntegra, bondadosa.

Por último, el hombre empieza en la vida material y terrena un camino de perfección y un ansia de felicidad que solo halla término en la trascendencia, donde recibirá la bienaventuranza divina como consecución plena de la felicidad añorada, en unión con el ser que "es el bien infinito, inmutable, seguro y gozosísimo, para quien lo alcanza. La educación perfecciona para la vida de Gracia, para la trascendencia. En Dios encuentra el hombre su perfección y su bien absolutos" (Beuchot, 2004, p. 192).

La mentalidad eficacista, materialista, egótica de nuestro superficialismo sensualista contemporáneo, vendió el espíritu humano a las finalidades del placer, el emotivismo acrítico, el lucro y el enriquecimiento a toda costa. Santo Tomás juzgaría todos esos planos que afectan la voluntad humana como pasiones que impiden la libertad y la consecución racional del bien por medio de la voluntad. Si hemos de progresar, el ascenso humano debe ser de una comunidad de hermanos, debe ser hacia una sociedad realmente justa, debe ser hacia valores más personalizantes, más trascendentales que el sensual, económico o partidista. Educar en valores, educar a la persona virtuosa, significa progresar en la promoción de todos los ciudadanos, más allá de las distinciones de credo, sexo, clase, opción política y posición económica.

La dinámica ascensional se calibra con la pregunta por la perfección: ¿Puede aspirarse a la integralidad en un país de niños nacidos de adolescentes que jugaban al amor? ¿Con un altísimo índice de pobreza, y un alarmante índice de desnutrición, es realista aspirar a formar integralmente? ¿Sin mínimos de nutrición y salud podremos proponer educación de la razón, del arte y del espíritu? ¿Cuáles son nuestras mediocridades? ¿Qué procesos nos ganan la autonomía, el 
bienestar, la libertad, el respeto? ¿Qué capacidades aún no han sido descubiertas y motivadas al desarrollo?

La educación considerada como proceso antropológico, nunca ha sido más requerida que en nuestro momento actual. Rescatar la integralidad, insistir en lo ético, ejercer la crítica para una comprensión de la realidad política, formar para acordar y actuar, enseñar a liderar, proponer y gestionar; todas estas misiones formativas, deben regalarnos una perspectiva humana que rescate nuestra conciencia histórica, tan maltrecha y herida por demasiados fenómenos de violencia y sin sentido impropios de la dignidad y la libertad del hombre. Así contribuiremos a forjar las comunidades tolerantes y respetuosas en las que se puede realizar los anhelos de justicia y paz. 


\section{Referencias}

Aquino, S. T. (2001). Suma de teología. Tomo I. Madrid: BAC.

(1989). Suma de teología. Tomo II. Madrid: BAC.

Aquino, S. T. (2007). De Veritate, q.11, a. 1. Revista eléctrónica mensual del Instituto Santo Tomás. e-aquinas, pp. 1-17. En: www.e-aquinas.net/epoca1/si-el-hombre-puedeensenar/1183451941.pdf+\&cd=3\&hl=es\&ct=clnk\&gl=co. Recuperado: 12/07/2015

Aristóteles. (1894/1993). Ética nicomáquea. Ética eudemia. Madrid: Gredos.

Beauchot, M. (2004). Introducción a la filosofía de Santo Tomás de Aquino. Salamanca: San Esteban.

Bowen, J. (1992). Historia de la educación occidental. Tomo II, La civilización de Europa. Barcelona: Herder.

Copleston, F. (1960). El pensamiento de Santo Tomás. México: Fondo de Cultura Económica.

\section{Ariel.}

. (2004). Historia de la filosofía. Vol. 2: De San Agustín a Escoto. Barcelona:

Díaz, C. (2002). Qué es el personalismo comunitario. Madrid: Ediciones Mounier.

Gilson, E. (1952/1999). La filosofía en la edad media. Madrid: Gredos.

Hernanz, F. (1945). Santo Tomás y la educación. Revista Cristiandad, 23, 104-107.

Martínez, E. (2002). Persona y educación en Santo Tomás de Aquino. Madrid: Fundación universitaria española.

. (2004). Ser y educar. Fundamentos de pedagogía tomista. Bogotá: Universidad Santo Tomás.

Millán-Puelles, A. (1989). La formación de la personalidad humana. Madrid: Rialp.

Restrepo, A. (2009). Tomismo. Notas pro-manuscritas de la Maestría en educación. Bogotá: Ediciones de la U. Santo Tomás.

Reyes, J. (2010). Hombre: Ser y conocer. Cúcuta: Copicentro impresores. 